\title{
ORIGEM, RAMIFICAÇÃO E DISTRIBUIÇÃO DA ARTÉRIA CELÍACA EM CANÁRIOS-DA-TERRA (Sicalis flaveola)
}

\section{ORIGIN, RAMIFICATION, AND DISTRIBUTION OF THE CELIAC ARTERY IN SAFFRON FINCH (Sicalis flaveola)}

\author{
Anne Cristine Oliveira Barbosa ${ }^{1}$ \\ Marcelle Santos Lemos ${ }^{1}$ \\ Cibele Geeverghese ${ }^{1}$ \\ Gabriel Batista Oliveira Borges ${ }^{1}$ \\ Marcelo Ismar Santana ${ }^{1^{*}}$
}

${ }^{1}$ Faculdade de Agronomia e Medicina Veterinária, Universidade de Brasília, Brasília, DF, Brasil.

*Autor para correspondência - misantana@unb.br

\section{Resumo}

A origem e a distribuição da artéria celíaca de trinta canários da terra (Sicalis flaveola) foram descritas por meio do preenchimento do sistema arterial com solução de látex corado e fixação em solução de formol, para posterior dissecação de seus ramos colaterais. A artéria celíaca originouse do ramo descendente da aorta, seu primeiro ramo visceral, direcionada para o antímero direito da cavidade celomática. Após emissão das artérias proventricular dorsal e esplênica, a artéria celíaca bifurcou-se em ramos esquerdo e direito: em todos os canários dissecados, o ramo esquerdo originou a artéria proventricular ventral a partir de sua face esquerda e as artérias gastroduodenal e gástrica ventral pela sua face direita, para em seguida continuar como artéria gástrica esquerda, seu ramo terminal. Em todas as aves, a artéria hepática direita foi o primeiro colateral originado na face direita do ramo direito, para em seguida originar, na face oposta, as artérias gástrica direita e ileal, continuando-se imediatamente como pancreaticoduodenal, seu ramo terminal. A partir desse estudo, concluiu-se que o canário da terra segue o mesmo padrão proposto quanto à origem e distribuição da artéria celíaca para aves dos gêneros Gallus, Anas, Columba, Crax, Mitu e Amazona. Palavras-chave: artéria celíaca; Canário da terra; distribuição; origem; Sicalis flaveola.

\begin{abstract}
This research was performed aiming to describe the origin and the distribution of the celiac artery in 30 Saffron finches (Sicalis flaveola), through the completion of their circulatory system with an aqueous solution of colored latex, followed by fixation in formol for later dissection of the collateral branches. The celiac artery originated from the descending branch of the aorta, representing its first visceral branch, and was directed to the right antimere of the coelomic cavity. After issuance of the proventricular dorsal artery and the splenic artery, the celiac bifurcated in left and right branches: In all observed finches the left branch originated the proventricular ventral artery, from its left side, and the gastroduodenal and ventral gastric arteries from its right side, in order to continue as the left gastric artery characterizing it as a terminal branch; from its right side, the right branch issued, as first collateral, the right hepatic artery in all observed birds, to originate, from its opposite side, the right gastric and ileal, immediately continuing as pancreaticoduodenal, its terminal branch. From this study, we concluded that the Saffron finch follows the same pattern regarding the origin and distribution of the celiac artery proposed for birds of the genus Gallus, Anas, Columba, Crax, Mitu, and Amazona.
\end{abstract}


Keywords: celiac artery; distribution; origin; Saffron finch; Sicalis flaveola.

Enviado em: 31 março de 2015

Aceito em: 06 abril de 2016

\section{Introdução}

Devido à cultura popular, várias espécies de aves brasileiras ainda são consideradas de gaiola, um hábito perpetuado sem controle e que tem causado enorme prejuízo para o meio ambiente, já que a ideia da população é de que a diversidade de nossa fauna não tem fim ${ }^{(1)}$. Infelizmente, esta mentalidade gerou a situação atual, em que se veem poucas aves silvestres voando em nossas fazendas, chácaras e áreas protegidas, pois o comércio ilegal gera uma renda expressiva, pois alguns exemplares chegam a valer verdadeiras fortunas, como os curiós (Sporophila angolensis) e os canários da terra (Sicalis flaveola) ${ }^{(2)}$. Por isto, torna-se importante a adoção de políticas de preservação da fauna, bem como de estímulo à criação de aves silvestres em cativeiro, na tentativa de desenvolver seu comércio legal, diminuindo consequentemente o tráfico ${ }^{(3)}$. Além disso, a criação de aves silvestres em cativeiro propicia a realização de estudos dentro das diferentes áreas do conhecimento, diminuindo assim a carência de informações científicas a respeito destes animais, além de permitir observar um número suficiente, através de análises estatísticas precisas ${ }^{(4)}$.

Levando-se em conta a grande demanda clínico-cirúrgica deste grupo de animais, médicos veterinários necessitam conhecer muito bem sua anatomia, com o intuito de facilitar o manejo e consequentemente a coleta de amostras laboratoriais, além da administração de medicamentos e procedimentos radiológicos e cirúrgicos ou durante a necropsia ${ }^{(5)}$. Desta forma, o estudo objetivou avaliar macroscopicamente a origem e a distribuição da artéria celíaca e de seus ramos em canários da terra (Sicalis flaveola), visando à comparação com outras espécies de aves domésticas e silvestres.

\section{Material e Métodos}

Foram utilizados 30 canários da terra (Sicalis flaveola), todos adultos e machos, doados pelo CETAS - DF (registro 1/53/930021-5), após óbito motivado por causas naturais. O protocolo experimental deste trabalho foi aprovado pelo Comitê de Ética no Uso Animal do Instituto de Ciências Biológicas (CEUA/IB) da Universidade de Brasília (protocolo 47173/2010).

Para destacar o sistema circulatório, as aves tiveram a porção cranial do celoma aberta, por meio de secção longitudinal do osso esterno e da respectiva musculatura peitoral até seu terço médio, o que permitiu o isolamento e a canulação do tronco braquiocefálico esquerdo com dispositivo intravascular flexível 16Gx2" (Jelco ${ }^{\circledR}$ ), para injeção de solução aquosa de látex a 50\% (Artecola $\left.{ }^{\circledR}\right)$ corada com corante a base de água (Suvinil ${ }^{\circledR}$ ), através de pressão manual.

As aves foram fixadas em solução aquosa de formol a $10 \%\left(\mathrm{Chemco}^{\circledR}\right)$ e mantidas em recipientes adequados, submersas na mesma solução, por um período mínimo de 48 horas, para finalização do processo de fixação e polimerização do látex. Após este período, procedeu-se à dissecação do tronco celíaco e seus ramos colaterais, com o auxílio de pinças anatômicas histológicas de

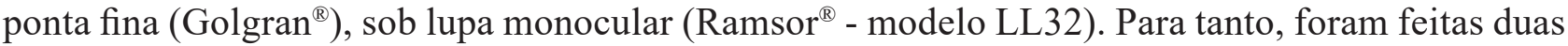
incisões longitudinais nos gradis costais contralaterais, como no terço disto-caudal da musculatura peitoral, expondo assim o celoma.

Os modelos de distribuição de cada exemplar foram transferidos esquematicamente para fichas individuais, onde foram registradas as distribuições configuradas pela artéria celíaca (Figura 01). A denominação das artérias teve como base os termos determinados pela Nomina Anatômica Aviária $^{(6)}$. 


\section{Resultados}

Em todos os exemplares, a artéria celíaca originou-se da aorta descendente, próxima ao terço médio do proventrículo, representando seu primeiro ramo visceral, direcionado para o antímero direito do celoma. Em 20 exemplares (67\%), a artéria celíaca emitiu a artéria proventricular dorsal como primeiro ramo colateral, com uma artéria esplênica em sequência, enquanto em outros 10 canários $(33 \%)$, duas artérias esplênicas foram os primeiros ramos, sendo a artéria proventricular dorsal o segundo.

Após a emissão dos ramos citados, a artéria celíaca bifurcou-se, próximo ao fígado, em ramos esquerdo e direito. Em todos os canários dissecados, o ramo esquerdo originou a artéria proventricular ventral a partir de sua face esquerda e, pela face direita, as artérias gastroduodenal, gástrica ventral e um ramo pericárdico (3,33\% dos casos), para, em seguida, enviar a artéria hepática esquerda e continuar como artéria gástrica esquerda, seu ramo terminal. De sua face direita, o ramo direito emitiu como primeiro colateral a artéria hepática direita em todas as aves observadas, para em seguida originar da face oposta as artérias gástrica direita e ileal, continuando-se imediatamente como artéria pancreaticoduodenal, seu ramo terminal.

Quanto à ordenação das artérias oriundas da artéria celíaca e de seus ramos esquerdo e direito, foi observado que as artérias estavam direcionadas para órgãos específicos como o esôfago, o baço, o proventículo, o ventrículo, os lobos hepáticos direito e esquerdo, a junção pilórica, o íleo e o pâncreas, que se comportaram da seguinte maneira (Figura 01):

Artéria proventricular dorsal: Nos 30 exemplares, teve como destino a superfície dorsal do proventrículo e esôfago, distribuindo-se com a emissão de dois (64\%) a três ramos (36\%).

Artéria esplênica: Independentemente do número de artérias originadas, uma (67\%) ou duas (33\%), em todos os casos tiveram como destino o hilo esplênico.

Artéria proventricular ventral: Teve como destino, em todos exemplares estudados, a superfície ventral do proventrículo e esôfago, enviando de um (40\%) a dois ramos (60\%). Ainda, emitiu em um exemplar $(3,33 \%)$ um ramo pericárdico direcionado para esôfago e ventrículo.

Artéria hepática esquerda: Em todas as aves dissecadas foi direcionada para a porta hepática, sendo responsável pela irrigação do lobo hepático esquerdo.

Artéria gastroduodenal: Responsável pela irrigação da face direita do ventrículo e junção pilórica em $100 \%$ dos canários, distribuindo três $(77 \%)$ ou quatro $(23 \%)$ pequenos ramos ao longo do seu percurso.

Artéria gástrica ventral: Em todas as aves estudadas, corria ao longo da face ventral do ventrículo, onde termina, emitindo de dois $(57 \%)$ a três $(43 \%)$ pequenos ramos.

Artéria gástrica esquerda: Em todos exemplares estudados, passou da margem dorsal do ventrículo para a face lateral esquerda, terminando junto à extremidade caudal do ventrículo. Em seu trajeto emitiu de três $(54 \%)$ a quatro $(46 \%)$ pequenos ramos.

Artéria hepática direita: Responsável pela irrigação do lobo hepático direito em todos os canários dissecados, sendo direcionada para a porta hepática. Nos dez exemplares que apresentaram vesícula biliar (33\%), a artéria hepática direita emitiu um delgado ramo cístico, distribuído pelo ducto cisticoentérico e corpo da vesícula.

Artéria gástrica direita: Em todos os exemplares, foi responsável pela irrigação da face lateral direita do ventrículo, enviando em seu trajeto de um (30\%) a dois (70\%) pequenos ramos, além de emitir um ramo pilórico à junção pilórica em todos exemplares.

Artéria ileal: Irrigou o segmento cranial do íleo em 100\% dos exemplares, distribuindo-se com a 
emissão de dois $(77 \%)$ ou três $(23 \%)$ ramos.

Artéria pancreaticoduodenal: Em todos os exemplares dissecados, irrigou o pâncreas e a flexura duodenal, com a emissão de cinco (17\%) a seis $(83 \%)$ ramos.

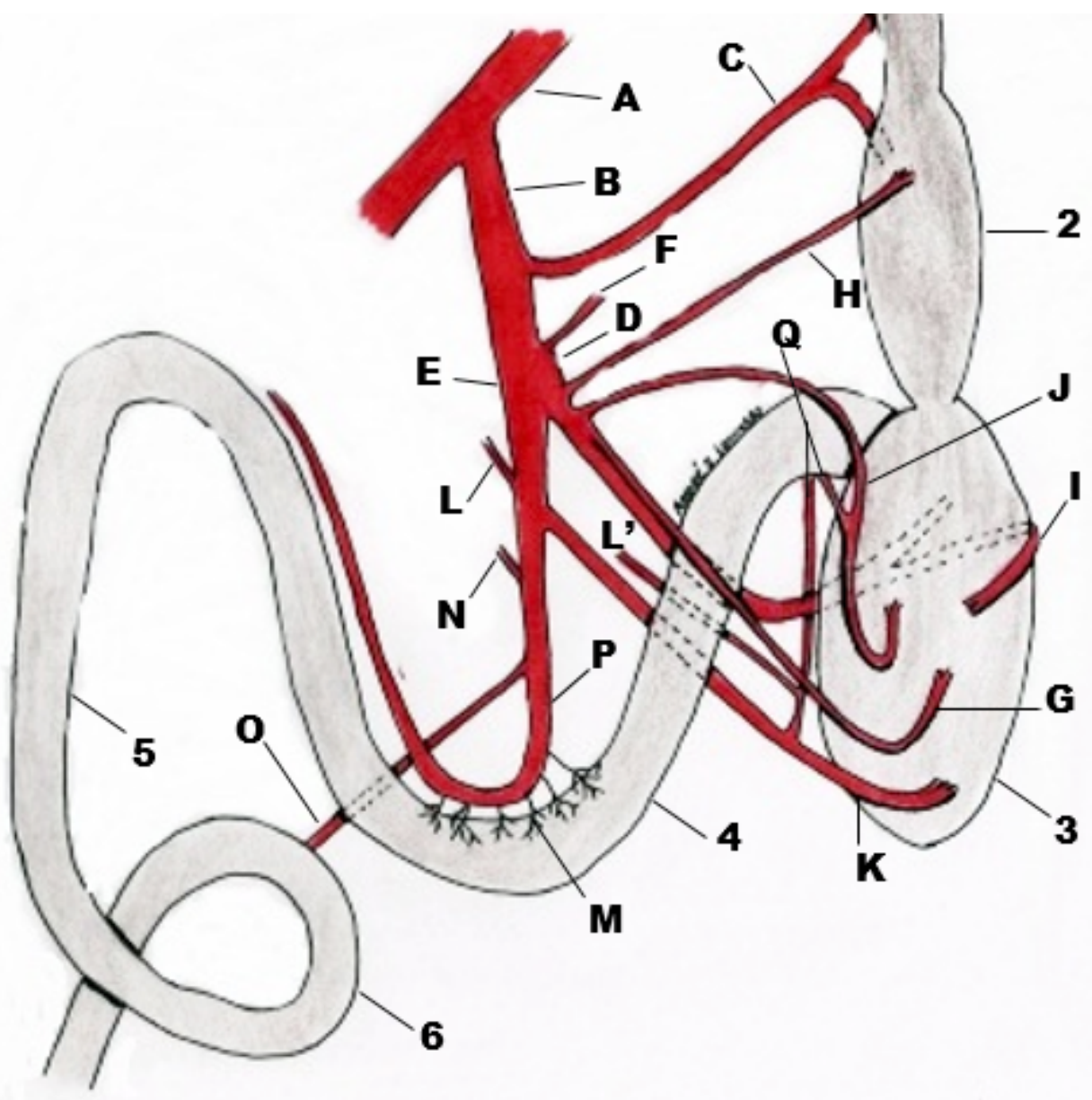

Figura 01. Representação esquemática da origem, ramificação e distribuição da artéria celíaca em zanários da terra (Sicalis flaveola). A - Aorta descendente abdominal; B - Artéria Celíaca; C Artéria proventricular dorsal; D - Ramo esquerdo da artéria celíaca; E - Ramo direito da artéria zelíaca; F - Artéria esplênica; G - Artéria gástrica ventral; H - Artéria proventricular ventral; I Artéria gástrica esquerda; J - Artéria gastroduodenal; $\mathrm{K}$ - Artéria gástrica direita; L - Artéria hepática direita; L' - Artéria Hepática esquerda; M - Ramos para a flexura duodenal; N - Ramo para o pâncreas; O - Artéria ileal; P - Artéria pancreaticoduodenal; Q - Ramos pilóricos; 1 Esôfago; 2 - Proventrículo; 3 -Ventrículo; 4-Duodeno; 5 - Jejuno; 6 -Íleo.

\section{Discussão}

Neste trabalho, observou-se que a forma de distribuição da artéria celíaca no canário da terra possui um padrão similar ao encontrado para o papagaio verdadeiro (Amazona aestiva) $)^{(7)}$ e os mutuns (Crax fasciolata e Mitu tuberosa) ${ }^{(8)}$, além de aves domésticas como o pato doméstico (Anas platyrhynchos $)^{(9)}$, 
linhagens comerciais do gênero Gallus ${ }^{(10)}$ e o pombo doméstico (Columba livia) ${ }^{(11)}$, já que essa artéria originou-se a partir da aorta descendente abdominal em sua porção ventral, no nível do proventrículo, enviando ramos diretamente para o proventrículo e o baço.

De maneira geral, a artéria celíaca distribui-se para a porção final do esôfago, pró-ventrículo, ventrículo, duodeno e porção final do jejuno-íleo, além do fígado, pâncreas e baço ${ }^{(11,12,13)}$. Estudos com diferentes aves domésticas de interesse comercial ${ }^{(14)}$ e aves silvestres ${ }^{(7,8,12,13)}$, mostraram que a artéria celíaca emitiu como seu primeiro ramo a artéria proventricular dorsal, de forma similar ao encontrado em $70 \%$ dos canários dissecados, enquanto os outros 30\% restantes tiveram a artéria esofágica como o seu primeiro ramo ${ }^{(10,15,16)}$. Deve-se relatar que na amostra analisada não foram encontrados ramos ou artérias esofágicas oriundos do tronco celíaco, o que vai de encontro às descrições de Silva Neto et al. ${ }^{(13)}$ a respeito do tucano de bico verde (Ramphastos dicolorus), cujo esôfago é irrigado exclusivamente pelas artérias proventriculares. Talvez, esta característica anatômica possa estar relacionada ao pequeno comprimento observado para o tronco celíaco dos canários, bem como pela grande proximidade com o proventrículo ${ }^{(16)}$.

Aycan e Duzler ${ }^{(15)}$ estudaram a coruja eurasiana (Bubo bubo) e reportaram que a artéria celíaca não apresentou divisão em ramos esquerdo e direito, sendo, portanto, formada por um tronco único que emite as artérias proventricular dorsal e ventral, esplênica e gastroduodenal. No entanto, como observado neste trabalho e para a maioria dos gêneros de aves ${ }^{(7,12,16)}$, a ausência da divisão do tronco celíaco em dois ramos de igual calibre não representa o padrão de ramificação da artéria celíaca, podendo ser, no caso do estudo citado, uma variação anatômica particular da coruja eurasiana ${ }^{(15)} \mathrm{em}$ função do grande volume alcançado pelo seu estômago, possivelmente pelo hábito de engolirem, de uma só vez, presas inteiras e de porte avantajado.

$\mathrm{O}$ ventrículo teve como fonte de irrigação as artérias gástrica ventral, gástrica esquerda e gástrica direita, característica semelhante à encontrada em papagaios verdadeiros ${ }^{(7)}$, $\operatorname{mutuns}^{(8)}$, tucano de bico verde $^{(13)}$ e para a maioria das linhagens comerciais do gênero Gallus já estudadas ${ }^{(10)}$, mas com a ausência da artéria gástrica dorsal, comumente encontrada nos gêneros citados ${ }^{(7)}$. Esta ausência nos canários dissecados, provavelmente, pode estar relacionada com a área de irrigação da artéria gástrica esquerda, que em seu trajeto enviou pequenos ramos para a superfície dorsal do ventrículo.

A artéria gastroduodenal também foi participante da irrigação do ventrículo, que nos canários estudados e nos tucanos de bico verde ${ }^{(13)}$, foi emitida pelo ramo esquerdo, sendo esta uma particularidade destas espécies, pois para outros gêneros de aves ${ }^{(10,16)}$, quando aparece, geralmente é originado pelo ramo direito da artéria celíaca, característica que não gera influência em sua área de irrigação.

Outro aspecto diferencial encontrado para a espécie estudada diz respeito à irrigação da junção pilórica que, da mesma forma que a observada no tucano de bico verde ${ }^{(13)}$, foi feita por ramos pilóricos oriundos da artéria gástrica direita, ao invés de diretamente do ramo direito da artéria celíaca, como no papagaio verdadeiro ${ }^{(7)}$, ou diretamente dos ramos direito e esquerdo como descrito para os pombos domésticos ${ }^{(11)}$. Provavelmente, esta variação representa uma característica particular dos canários e que não deve influenciar na fisiologia deste segmento do canal alimentar.

Da mesma forma que no Mitu tuberosa ${ }^{(8)}$, em um exemplar de Sicalis flaveola a artéria proventricular ventral emitiu um ramo pericárdico, irrigando o esôfago e o ventrículo, coincidindo com as descrições de Rafael et al. ${ }^{(17)}$, Miranda et al. ${ }^{(18)}$ e Gonçalves et al. ${ }^{(8)}$. Além disso, pela baixa ocorrência relatada, sua presença pode ser caracterizada como variação individual para as espécies citadas ${ }^{(7)}$.

A espécie estudada também não apresentou individualmente a artéria duodenojejunal, descrita para outras aves ${ }^{(8,13)}$ e sim uma artéria gastroduodenal, variação que pode ter relação direta com a área de irrigação da artéria mesentérica cranial ${ }^{(19)}$.

Nos dez exemplares de canários da terra que apresentaram vesícula biliar, estas receberam um ramo cístico oriundo da artéria hepática direita, característica semelhante à observada para os gêneros Gallus $^{(14)}$ e Buteo $^{(16)}$. A ausência de vesícula biliar na maioria das aves estudadas assemelha-se às descrições encontradas para papagaios verdadeiros ${ }^{(7)}$ e pombos domésticos ${ }^{(11)}$. 
Nos canários da terra, o baço foi irrigado exclusivamente pelas artérias esplênicas originadas do tronco da artéria celíaca ${ }^{(7,8,16)}$, fato não coincidente com os relatos feitos para as linhagens comerciais do gênero Gallus, que se originaram principalmente do ramo direito da artéria celíaca ${ }^{(17)}$ ou de ambos os ramos da celíaca ${ }^{(18)}$.

\section{Conclusões}

O arranjo apresentado pela artéria celíaca e seus ramos colaterais em Sicalis flaveola mostrou uma distribuição muito similar ao encontrado para aves domésticas (Gallus gallus domesticus, Anas platyrhynchos e Columba livia) e silvestres (Crax fasciolata, Mitu tuberosa e Amazona aestiva), apesar de não existir um padrão para o número de ramos emitidos por ela.

\section{Referências}

1. Brasil. Ibama. Sistema de Cadastro de Criadores Amadoristas de Passeriformes. Disponível em: http://servicos.ibama.gov.br/index.php/manual-do-sistema/131-cadastro-de-criadores-amadoristas-depasseriformes-sispass?format $=$ pdf

2. Pereira GA, Brito MT. Diversidade de aves brasileiras comercializadas nas feiras livres da região metropolitana do Recife, Pernambuco. AO. 2005;126:14-20. Disponível em: http://www.ao.com.br/download/glauco.pdf

3. Seixas GHF. Projeto papagaio-verdadeiro (Amazona aestiva): manejo e conservação no Pantanal e Cerrado de Mato Grosso do Sul, Brasil. Pub. Vet. 2007;1(8):1-8. Disponível em: https://papagaioverdadeiro.wordpress. $\underline{\text { com/artigos }}$

4. Silveira LF. Pela criação comercial de aves brasileiras. Cães e Cia. 2013;408:48-50. Disponível em: http:// www.ib.usp.br/ lfsilveira/pdf/a_2013_ceccriacao.pdf

5. Miller RE, Fowler, ME. Fowler's Zoo and wild animal medicine: corrent therapy. 7th ed. St. Louis: Elsevier Saunders; 2012. 669p. Inglês.

6. Baumel JJ. Handbook of avian anatomy: nomina anatomica avium. 2nd ed. Cambridge: Nuttall Ornithological Club; 1993. p. 779. Inglês.

7. Gonçalves ES, Santana MI, Zancan FT, Pinto ABF, Lima EMM. Distribuição configurada pela artéria celíaca em papagaios verdadeiros (Amazona aestiva). Arq. Bras. Med. Vet. Zootec. 2011;63(5):1141-1148. Disponível em: http://dx.doi.org/10.1590/S0102-09352011000500014

8. Gonçalves, ES, Santana MIS, Lima EMM, Silva FOC, Severino RS, Drummond SS. Origem e distribuição da artéria celíaca em mutuns dos gêneros Crax e Mitu. Ars Vet. 2010;26(2):88-94. Disponível em: http://www. arsveterinaria.org.br/index.php/ars/article/view/283/230

9. Resende GGN, Silva FOC, Vasconcelos BG, Campos DB, Campos AB, Ferreira FA, Rinaldi FCQ. Origem e distribuição da artéria celíaca em marrecos (Anas platyrhynchos platyrhynchos). Vet. Not. 2010;16(1):57-61. Disponível em: http://www.seer.ufu.br/index.php/vetnot/article/view/18957/10215

10. Carvalho FS, Severino RS, Rinaldi FCQ, Silva TA, Pereira SA. Origem e distribuição da artéria celíaca em aves (Gallus gallus domesticus) da linhagem Cobb Avian 48. Vet. Not. 2011;17(2):148-154. Disponível em: http://www.seer.ufu.br/index.php/vetnot/article/viewFile/19004/12329

11. Geeverghese C, Barbosa ACO, Lemos MS, Borges GBO, Santana, MIS, Lima, EMM. Descrição da artéria 
celíaca em pombos domésticos (Columba livia). Biotemas. 2012;25(2):125-131. Disponível em: https:// periodicos.ufsc.br/index.php/biotemas/article/viewFile/2175-7925.2012v25n2p125/21816

12. Vasconcelos BG, Silva FOC, Miranda RL, Pereira CCH, Santos AC, Miglino MA Origem e distribuição da artéria celíaca de avestruzes (Struthio camelus). Ci. Anim. Bras. 2012;13(1):108-114. Disponível em: http:// www.revistas.ufg.br/index.php/vet/article/view/15520/10598

13. Silva Neto OJ, Rosa MCB, Bonifácio TMM, Pinto ABF, Guimarães CSO, Guimarães GC. Origem, ramificação e distribuição da artéria celíaca no tucano de bico verde (Ramphastos dicolorus, Linnaeus, 1766). Pesq. Vet. Bras. 2013;33(3):399-404. Disponível em: http://www.scielo.br/pdf/pvb/v33n3/21.pdf

14. Kuru N. Macroanatomic investigations on the course and distribution of the celiac artery in domestic fowl (Gallus gallus domesticus). Sci. Res. Essays. 2010;5(23):3585-3591. Disponível em: http://www. academicjournals.org/SRE. Inglês.

15. Aycan K, Duzler A. The anatomy of celiac artery in the eagle owl (Bubo bubo). Ankara Univ. Vet. Fak. 2000;47:319-323. Disponível em: http://dergiler.ankara.edu.tr/dergiler/11/549/6783.pdf. Inglês.

16. Haligur A, Duzler A. Course and branch of the celiac artery in the red falcon (Buteo rufinus). Vet. Med. Czech. 2010; 55(2):79-86. Disponível em: http://vri.cz/docs/vetmed/55-2-79.pdf. Inglês.

17. Rafael ELS, Silva FOC, Severino RS, Drummond, SS., Bombonato, PP, Peres, RFG. Origem e distribuição da artéria celíaca em aves (Gallus gallus Linnaeus 1758) da linhagem Arbor Acres. Biosci. J. 2005;21(3):5560. http://www.seer.ufu.br/index.php/biosciencejournal/article/view/6607/4340

18. Miranda RL, Silva FOC, Severino RS, Drummond, SS, Sola, MC, Mendonça EP, Faria AB. Origem e distribuição da artéria celíaca em aves (Gallus gallus) poedeiras da linhagem Dekalb White. Vet. Not. 2006;12(2):41-46. Disponível em: http://www.seer.ufu.br/index.php/vetnot/article/view/18808/10125

19. Pinto MRA, Ribeiro AACM, Souza WM. Os arranjos configurados pela artéria celíaca no pato doméstico (Cairina moshata). Braz. J. Vet. Res. An. Sci. 1998;35(3):103-106. Disponível em: http://www.scielo.br/pdf/ bjvras/v35n3/35n3a01.pdf 